九州大学学術情報リポジトリ

Kyushu University Institutional Repository

\title{
Electron Density Measurement Inside a Hall Thruster Using Microwave Interferometry
}

\section{Kuwabara, Naoya}

Department of Advanced Energy Engineering Science, Interdisciplinary Graduate School of

Engineering Sciences, Kyushu University : Master's Program

Chono, Masatoshi

Department of Advanced Energy Engineering Science, Interdisciplinary Graduate School of Engineering Sciences, Kyushu University : Master's Program

Yamamoto, Naoji

Department of Advanced Energy Science and Engineering, Faculty of Engineering Sciences, Kyushu University : Professor

Kuwahara, Daisuke

College of Engineering, Chubu University : Senior Assistant Professor

http://hdl. handle. net/2324/4371074

出版情報: Journal of propulsion and power, 2021-03-04. American Institute of Aeronautics and Astronautics

バージョン：

権利関係 : 


\title{
Electron Density Measurement Inside a Hall Thruster Using Microwave Interferometry
}

\author{
Naoya Kuwabara, ${ }^{1}$ Masatoshi Chono ${ }^{2}$ and Naoji Yamamoto, ${ }^{3}$ \\ Kyushu University, Kasuga, Fukuoka, 816-8580, Japan \\ and \\ Daisuke Kuwahara $^{4}$ \\ Chubu University, Kasugai, Aichi, 487-8501, Japan
}

\section{Nomenclature}

$$
\begin{array}{ll}
B & =\text { magnetic flux density } \\
d & =\text { channel width } \\
e & =\text { elementary charge } \\
f & =\text { microwave frequency } \\
f_{\mathrm{p}} & =\text { plasma frequency } \\
I & =\text { in-phase component of signal } \\
I_{\mathrm{d}} & =\text { discharge current } \\
I_{\mathrm{ic}} & =\text { coil current } \\
m_{\mathrm{e}} & =\text { electron mass } \\
N & =\text { refractive index of plasma } \\
Q & =\text { quadrature component of signal } \\
n_{\mathrm{e}} & =\text { line-averaged electron number density } \\
V_{\mathrm{d}} & =\text { discharge voltage } \\
\varepsilon_{0} & =\text { permittivity of vacuum } \\
\lambda & =\text { wavelength of microwave in vacuum }
\end{array}
$$

\footnotetext{
${ }^{1}$ Graduate student, Department of Advanced Energy Engineering Science, 6-1 Kasuga-kouen, Kasuga, Fukuoka 816-8580, Japan, kuwabara.naoya.174@s.kyushu-u.ac.jp

${ }^{2}$ Graduate student, Department of Advanced Energy Engineering Science, 6-1 Kasuga-kouen, Kasuga, Fukuoka 816-8580, Japan, c.masatoshi@gmail.com

${ }^{3}$ Professor, Department of Advanced Energy Engineering Science, 6-1 Kasuga-kouen, Kasuga, Fukuoka 816-8580, Japan, yamamoto@aees.kyushu-u.ac.jp and Member AIAA.

${ }^{4}$ Senior Assistant Professor, College of Engineering, 1200 Matsumoto-cho, Kasugai, Aichi, 487-8501, Japan, dkuwahara@ isc.chubu.ac.jp.
} 
$\phi \quad=\quad$ phase shift of microwave

\section{Introduction}

【 nderstanding the physics behind anomalous electron transport [1-4] will be crucial for development of Hall thrusters. A wide variety of theories has been developed to describe anomalous electron transport in Hall thrusters, but the problem remains unresolved. Hirakawa [5] and Janhunen [6] showed that azimuthal electric field fluctuation (azimuthal $\mathrm{MHz} \boldsymbol{E} \times \boldsymbol{B}$ drift instability) can lead to anomalous electron transport. Near wall conductivity [7, 8] and Reynolds stress [9] are also possible drivers of anomalous transport. Thus, as a first step to understanding anomalous electron transport, the nature of instabilities observed in Hall thrusters and the relationship between anomalous transport and electric field oscillations (deduced from number density fluctuations) should be investigated. As a first step, temporally resolved electron number density measurements inside the acceleration channel in the Hall thruster are needed.

There are many techniques for measuring the electron number density in Hall thrusters, including Langmuir probes [10], laser induced fluorescence (LIF) [11, 12], and laser Thomson scattering [13, 14]. However, MHz electron number density fluctuation inside the Hall thrusters cannot yet be measured without perturbation of the plasma; Langmuir probes disturb the thruster operation and the bandwidth of LIF [11, 12], the incoherent Thomson scattering technique [13] is insufficient to fully resolve $\mathrm{MHz}$ oscillations, and it is problematic to measure inside the plasma by collective Thomson scattering [14] due to the inaccessibility of the probe laser.

In this study, a method for temporally resolved plasma density measurement inside the acceleration channel of a Hall thruster using a $76 \mathrm{GHz}$ microwave interferometer is demonstrated. The microwave interferometer has already shown its usefulness for the measurement of electron number density fluctuation in other contexts [15-17]. It is a nonintrusive measurement technique, that is, it does not disturb the measured plasma, and it can offer wide bandwidth up to a few $\mathrm{MHz}$, which is sufficient to fully resolve $\mathrm{MHz}$ oscillation. Crucially, this measurement technique does not require any changes to the acceleration channel in magnetic layer type (SPT type) Hall thrusters, since the probe microwaves can pass through the acceleration channel, which is constructed of dielectric material (boron nitride (BN), borosil (BN-SiO2), and among other materials). Here, we show for the first time that the method can be extended to number density measurement inside Hall thrusters, supporting thruster development and contributing to a better understanding of anomalous electron transport. 


\section{Experiment}

\section{A. Microwave Interferometry}

Microwave interferometry can be used to estimate line-averaged electron number density along a propagation path from the phase difference between the transmitted wave propagating through the plasma and a reference wave propagating through the cable or vacuum [18].

A block diagram of the microwave interferometry system is shown in Fig. 1. The system uses a frequencymultiplied superheterodyne method with two oscillators [17]. The RF frequency is determined considering the cutoff electron density $\left(7.2 \times 10^{19} \mathrm{~m}^{-3}\right)$ and the illumination frequency limit of the measuring equipment. Pre-local oscillation (pre-LO, $19 \mathrm{GHz}$ ) waves are up-converted to $19.0275 \mathrm{GHz}$ (pre-radio frequency waves (pre-RF)) by mixing a 27.5 MHz signal in a mixer. The pre-RF waves are frequency multiplied up to $76.11 \mathrm{GHz}(\mathrm{RF})$ in a transmission module. The RF waves propagate through a waveguide, are emitted from an antenna, pass through the plasma, and are received by a receiving module. The RF waves and local oscillation waves (LO) are down-converted to intermediate frequency (IF) waves $(110 \mathrm{MHz})$, which contain phase shift information, in the receiving module. With quadruplers in the transmission and receiving modules [15], a coaxial cable transmission line can be used instead of a waveguide transmission line to transmit the waves from the wave source outside of the vacuum chamber to the transmission module and to the receiving module in the vacuum chamber.

The detected IF signal is output as $I(\cos \phi)$ and $Q(\sin \phi)$ signals by an orthogonal detector (IQ detector). Following Ref. 18, the phase shift $\phi$, which is calculated from the arc tangent value of $I$ and $Q$, is converted into lineaveraged electron number density $n_{\mathrm{e}}$ by Eqs. (1) to (3).

$$
\begin{gathered}
\phi=\frac{2 \pi d}{\lambda}(1-N) \\
N=\sqrt{1-\frac{f_{\mathrm{p}}^{2}}{f^{2}}} \\
f_{\mathrm{p}}=\frac{1}{2 \pi} \sqrt{\frac{n_{\mathrm{e}} e^{2}}{m_{\mathrm{e}} \varepsilon_{0}}}
\end{gathered}
$$

Here, assuming that the number density in the acceleration channel is uniform in the radial direction, and we calculate it under the condition that the plasma penetration length is the same as the channel width $d(15 \mathrm{~mm}$ in the present configuration). From Eqs. (1) to (3), the relational expression between $n_{\mathrm{e}}$ and $\phi$ can be obtained as shown in Eq. (4). 


$$
n_{\mathrm{e}}=\frac{4 \pi^{2} m_{\mathrm{e}} \varepsilon_{0} f^{2}}{e^{2}}\left\{1-\left(1-\frac{\lambda}{2 \pi d} \phi\right)^{2}\right\}
$$

The absolute electron number density is estimated from the phase difference before/after stopping thruster operation.

A schematic of the measurement region is shown in Fig. 2. The RF waves are transmitted through WR-12 wave guides $(3.10 \mathrm{~mm} \times 1.55 \mathrm{~mm})$, are emitted and pass through the channel wall and the plasma, and are received by a pyramidal horn-type antenna on the receiving module. The polarization direction (electric field vector) of the RF waves is the azimuthal direction of the Hall thruster. The RF antenna is set inside the inner acceleration channel wall and the receiving antenna is set outside the outer acceleration channel wall, with the two antennas facing each other at the 10 o'clock location as measured from the front of the Hall thruster. As shown in Fig. 2, the waveguide of the emitting antenna is bent toward the radial direction of the Hall thruster, and the end of the microwave outlet is set 3 $\mathrm{mm}$ upstream of the Hall thruster exit. The reception antenna has a pyramidal horn with a length of $19 \mathrm{~mm}$, and the aperture area is $8 \mathrm{~mm} \times 8 \mathrm{~mm}$. The aperture angle is $19.2^{\circ}$ in the electric field direction and $14.6^{\circ}$ in the magnetic field direction.

The analog bandwidth of the high-speed insulation module in the data recorder and the low pass filter cutoff frequency of IQ detector are $20 \mathrm{MHz}$ and 7.7 MHz, respectively. Therefore, the bandwidth of this system is $7.7 \mathrm{MHz}$. 


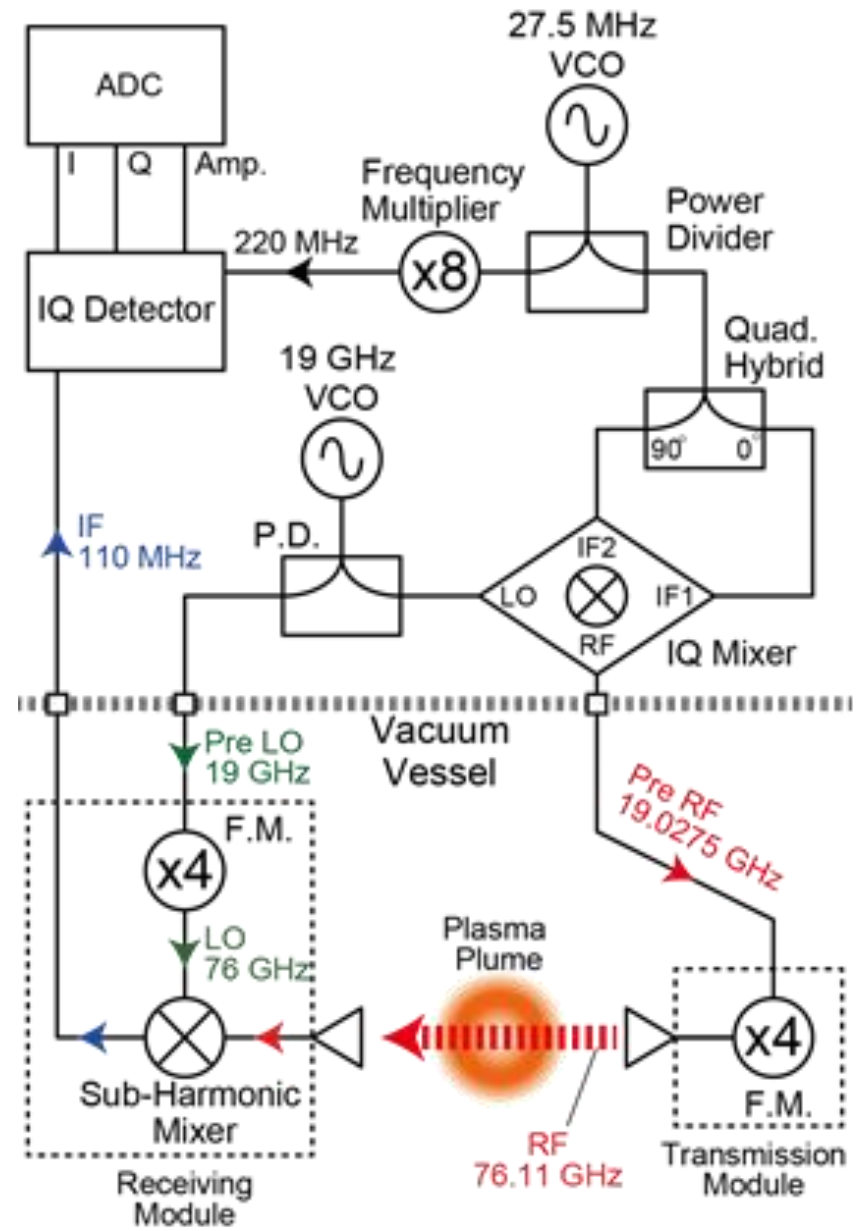

Fig. 1 Block diagram of microwave interferometry.

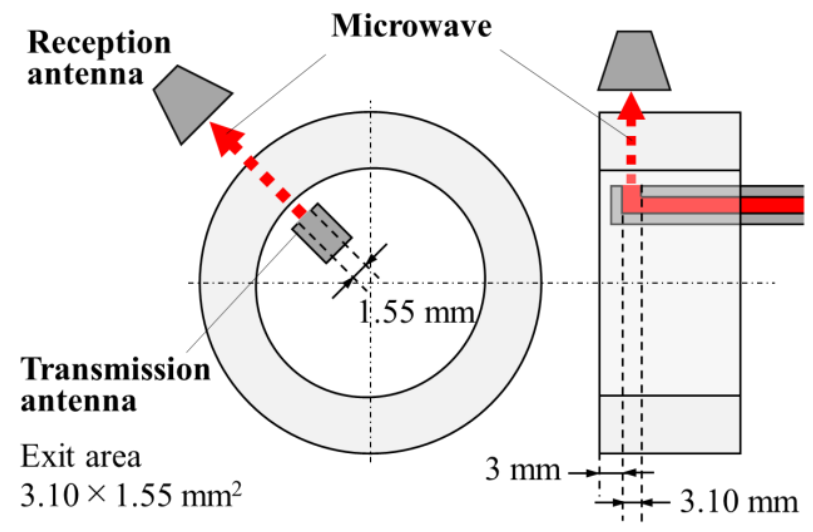

Fig. 2 Schematic of measurement region. 


\section{B. Hall Thruster}

The $600 \mathrm{~W}$ class magnetic layer-type Hall thruster used in this study was developed at Kyushu University. The outer dimensions of the thruster are $200 \mathrm{~mm} \times 200 \mathrm{~mm} \times 85 \mathrm{~mm}$. The acceleration channel is made of boron nitride, the inner and outer diameters of the acceleration channel are $61 \mathrm{~mm}$ and $91 \mathrm{~mm}$, respectively, and the inner and outer walls of the acceleration channel are $2 \mathrm{~mm}$ thick. The anode is set at $14 \mathrm{~mm}$ upstream of the thruster exit. The magnetic circuit is composed of five solenoid coils, one at the center of the thruster and the others outside the channel, so the magnetic field can be applied in the radial direction. The number of turns in each coil is the same, and the magnetic field strength is adjusted by changing the current to each coil. In order to insert the waveguides from the rear of the thruster into the space between the inner coil and the acceleration channel, there are 12 holes ( 8 mm diameter) on the back yoke. A hollow cathode is used as the electron source. The cathode is mounted on the thruster and tilted at 45 degrees toward the downstream direction. Xenon gas (purity 99.999\%) is used as the propellant gas, and the mass flow rates through the thruster and cathode are fixed at $2.72 \mathrm{mg} / \mathrm{s}$ and $0.27 \mathrm{mg} / \mathrm{s}$, respectively, using two mass flow controllers.

\section{Vacuum Facility}

The experiment was conducted in the $2.5 \mathrm{~m}$ diameter by $5.0 \mathrm{~m}$ long space science plasma chamber at the Institute of Space and Astronautical Science (ISAS) / Japan Aerospace Exploration Agency (JAXA). The pumping system is composed of a rotary pump, a mechanical booster pump, a turbo molecular pump (pumping speed 3,400 1/s on nitrogen) and two cryogenic pumps (pumping speed 22,000 1/s on nitrogen). The chamber baseline pressure is below $1 \times 10^{-5} \mathrm{~Pa}\left(<1 \times 10^{-9}\right.$ Torr $)$, and the pressure during thruster operation is below $1 \times 10^{-2} \mathrm{~Pa}\left(<1 \times 10^{-5}\right.$ Torr $)$.

\section{Results and Discussion}

Figure 3 shows the dependency of time-averaged electron number density and time-averaged discharge current on magnetic field strength (coil current). The time-averaged discharge current decreases with an increase in magnetic field strength until the coil current reaches $1.1 \mathrm{~A}$, and then it increases with an increase in coil current. The timeaveraged electron density shows almost the same tendency as the discharge current, except that it suddenly increases at a coil current of $1.5 \mathrm{~A}$, when the plume shape changes from a sharp shape to a rounded shape. The line-averaged number density is approximately $10^{18} \mathrm{~m}^{-3}$, which is in good agreement with other measurements in Hall thrusters [19]. The uncertainty is evaluated from two components: the standard error $\left(1.9 \times 10^{15} \mathrm{~m}^{-3}\right)$, and the uncertainty of thermal 
drift coefficient related to microwave transmitter components $\left(1 \times 10^{14} \mathrm{~m}^{-3} / \mathrm{s}\right)$. The uncertainty of averaged electron density is estimated as $3.5 \times 10^{16} \mathrm{~m}^{-3}$ at a coil current of $0.5 \mathrm{~A}$. The uncertainty of the discharge current is evaluated from standard error $\left(1.0 \times 10^{-2} \mathrm{~A}\right)$.

Figure 4 shows the time variation of the line-averaged electron density and the discharge current at a $V_{\mathrm{d}}$ of $300 \mathrm{~V}$ and $I_{\mathrm{ic}}$ of $0.75 \mathrm{~A}$. Considering the time scale of the measurement, the effect of the thermal drift on the relative electron number density can be almost ignored. The absolute value of the electron density is estimated by adding the relative electron number density to the time-averaged number density under the same conditions ( $V_{\mathrm{d}}$ of $300 \mathrm{~V}$ and $I_{\mathrm{ic}}$ of 0.75 A). As shown in Fig. 4, measured electron number density and discharge current fluctuate with the wave period of $0.07 \mathrm{~ms}$ (oscillation frequency of $15 \mathrm{kHz}$ ). The electron number density fluctuation is synchronized with discharge current oscillation with a phase lag. There is high frequency oscillation in both electron number density and discharge current, but they are not synchronized.

Figure 5 shows the fast Fourier transform (FFT) spectrum of the electron density and the discharge current from $1 \times 10^{3}$ to $5 \times 10^{6} \mathrm{~Hz}$. These spectra are calculated using the one-dimensional inverse discrete Fourier Transform (FFT) algorithm, using the numpy $\mathrm{ftt}$ module. The sampling rate is $100 \mathrm{MS} / \mathrm{s}$, and record length is $10 \mathrm{~ms}$. From the spectrum of thruster off (without plasma), the noise floor is estimated as $1 \times 10^{15} \mathrm{~m}^{-3}$ for frequencies above $100 \mathrm{kHz}$. As shown in Fig. 5, the largest oscillations in electron density and discharge current can be observed at $15 \mathrm{kHz}$. This is the socalled ionization oscillation or breathing mode oscillation [19-22], which corresponds to the $0.07 \mathrm{~ms}$ period oscillation described in Fig. 5. There is a clear peak at $200 \mathrm{kHz}$ in the discharge current oscillation, the so-called transit oscillation [8], although there is no clear peak in electron number density. Instead, a broadband oscillation (50 to $300 \mathrm{KHz}) \mathrm{of}$ irregular period was observed in the electron number density fluctuation. There is an oscillation with a broadband peak (width of $200 \mathrm{kHz}$ ) at approximately $1.5 \mathrm{MHz}$ in the electron density; this is the electron drift instability. Though this peak cannot be observed in the discharge current oscillation, a broadband peak from $500 \mathrm{kHz}$ to $2 \mathrm{MHz}$ can be observed. There are several narrow peaks from sub $\mathrm{MHz}$ to $\mathrm{MHz}$; these are derived from the oscillators (27.5 MHz voltage controlled oscillator (VCO) and $19 \mathrm{GHz}$ VCO). 


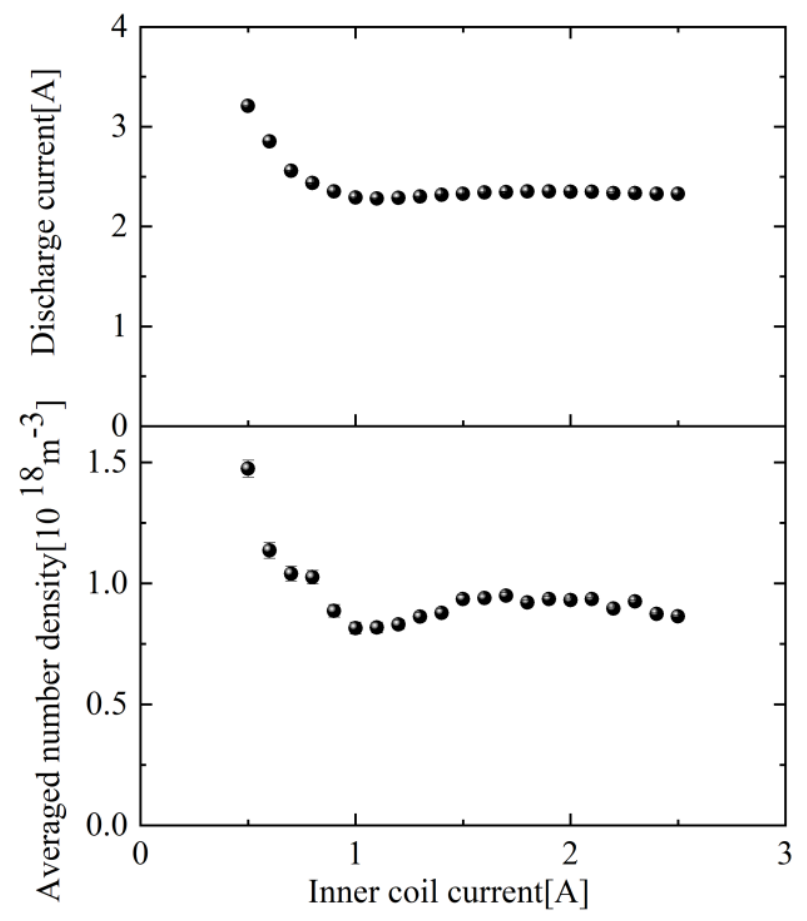

Fig. 3 Magnetic field dependency of electron density and discharge current.

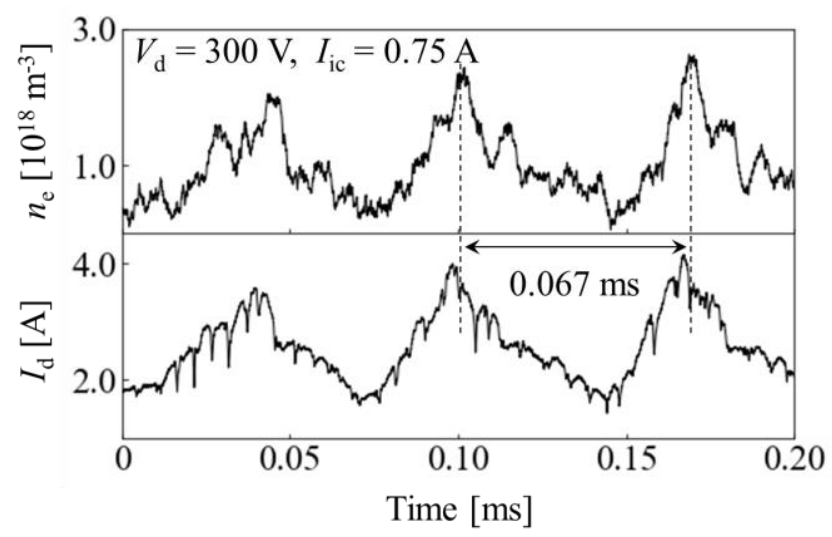

Fig. 4 Time variation of electron density and discharge current. 


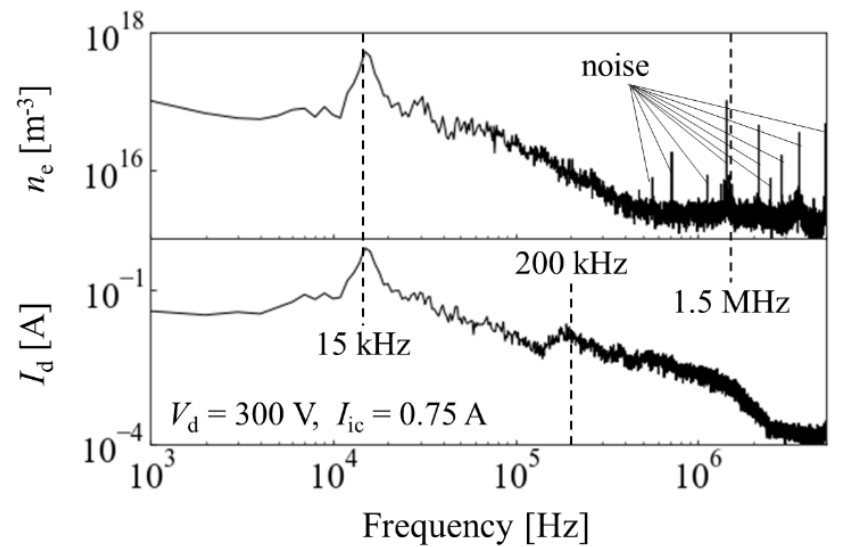

Fig. 5 FFT spectrum of electron density and discharge current.

\section{Conclusions}

Electron number density measurement inside the Hall thruster was demonstrated using a $76 \mathrm{GHz}$ microwave interferometry technique. The electron number density, which depends on the magnetic field strength, was found to be approximately $10^{18} \mathrm{~m}^{-3}$. Electron density fluctuations were observed at a frequency of $15 \mathrm{kHz}$, synchronized with the discharge current oscillation. Broadband electron number density fluctuations were also observed in the range of 50-300 kHz, and at approximately $1.5 \mathrm{MHz}$ (width of $200 \mathrm{kHz}$ ). This technique shows considerable promise, with the ability to reveal the quantitative relationship between electron number density fluctuations and electron transport, and it will contribute to the understanding of the physics behind anomalous electron transport in Hall thrusters.

\section{Acknowledgments}

The experiments were performed at the Space Plasma Laboratory of ISAS, JAXA. This work was supported by JSPS KAKENHI Grants JP18H03815, JP18KK0406 and JP19K14683.

\section{References}

[1] Lafleur, T., Baalrud, S. D., and Chabert, P., “Theory for the Anomalous Transport in Hall Effect Thrusters. I. Insights from Particle-in-cell Simulations," Physics of Plasmas, Vol. 23, No. 5, 9 May 2016, 053502.

https://doi.org/10.1063/1.4948495

[2] Lafleur, T., Baalrud, S. D., and Chabert, P., "Theory for the Anomalous Transport in Hall Effect Thrusters. II. Kinetic Model,” Physics of Plasmas, Vol. 23, No. 5, 9 May 2016, 053503. 
https://doi.org/10.1063/1.4948496

[3] Meezan, N. B., Hargus, Jr., W. A., and Cappelli, M. A., “Anomalous Electron Mobility in a Coaxial Hall Discharge Plasma,” Physical Review E, Vol. 63, No. 2, 24 Jan. 2001, 026410.

https://doi.org/10.1103/PhysRevE.63.026410

[4] Morozov, A. I., Esipchuk, Yu. V., Kapulkin, A. M., Nevrovskii, V. A., and Smirnov, V. A., “Azimuthally Asymmetric Modes and Anomalous Conductivity in Closed Electron Drift Accelerators," Soviet Physics Technical Physics, Vol. 18, Nov. 1973, pp. 615-620.

[5] Hirakawa, M., and Arakawa, Y. "Particle Simulation of Plasma in Electric Propulsion Thrusters," The Japan Society for Aeronautical and Space Sciences Vol.45 (1997), pp. 444-452, (in Japanese).

https://doi.org/10.2322/jjsass 1969.45.444

[6] Janhunen, S., Smolyakov, A., Chapurin, O., Sydorenko, D., Kaganovich, I., and Raitses, Y., "Nonlinear Structures and Anomalous Transport in Partially Magnetized Plasmas,” Physic of Plasma, Vol. 25, 011608, 2018

https://doi.org/10.1063/1.5001206

[7] Makowski, K., Peradzynski, Z., Kolanowski, M., Barral, S., Kurzyna, J., and Dudeck, M., "Near Wall Conductivity in Hall Thrusters. Cylindrical Geometry Effect,” International Electric Propulsion Conference, IEPC-2007-246, 2007.

[8] Choueiri, E. Y., "Plasma Oscillations in Hall Thrusters,” Physics of Plasmas, Vol. 8, No. 4, 22 Mar. 2001, pp. 1411-1426. https://doi.org/10.1063/1.1354644

[9] Cho, S., Hara, K., Watanabe, H., Kubota, K., and Yamashita, Y., "Investigation of Cross-Field Electron Transport in a 100-W Class Hall Thruster Using a Full Particle-in-Cell Simulation,” International Electric Propulsion Conference, IEPC-2019-718, 2019.

[10] Lobbia R. B. and Beal B. E., "Recommended Practice for Use of Langmuir Probes in Electric Propulsion Testing," Journal of Propulsion and Power, Vol. 33, No.3, 2017, pp. 566-581.

https://doi.org/10.2514/1.B35531

[11] Mazouffre, S., Gawron, D., and Sadeghi, N., “A Time-resolved Laser Induced Fluorescence Study on the Ion Velocity Distribution Function in a Hall Thruster After a Fast Current Disruption,” Physics of Plasmas, Vol. 16, No. 4, 16 Apr. 2009 , 043504.

https://doi.org/10.1063/1.3112704

[12] Chaplin, V. H., Lobbia, R. B., Lopez Ortega, A., Mikellides, I. G., Hofer, R. R., Polk, J. E. and Friss, A. J. , “Time-Resolved Ion Velocity Measurements In A High-Power Hall Thruster Using Laser-Induced Fluorescence With Transfer Function Averaging," Applied Physics Letters, Vol.116, 234107, 2020.

https://doi.org/10.1063/5.0007161 
[13] Tomita, K., Yamamoto, N., Yamasaki, N., Tsuru, T., Uchino, K., and Nakashima, H., “Thomson-Scattering Diagnostics of Plasmas Produced in Miniature Microwave Discharge Ion Engine,” Journal of Propulsion and Power, Vol. 26, No.2, 2012, pp. 381-384.

https://doi.org/10.2514/1.39145

[14] Tsikata, S., Honoréb, C., and Grésillonb, D., "Collective Thomson Scattering for Studying Plasma Instabilities In Electric Thrusters," Journal of Instrumentation Vol. 8, C10012, 2013.

https://doi.org/10.1088/1748-0221/8/10/C10012

[15] Kuwahara, D., Ito, N., Nagayama, Y., Tsuchiya, H., Yoshikawa, M., Kohagura, J., Yoshinaga, T., Yamaguchi, S., Kogi, Y., Mase, A., and Shinohara S., "Development of Local Oscillator Integrated Antenna Array for Microwave Imaging Diagnostics," Journal of Instruments, Vol. 10, 29 Dec. 2015, C12031.

https://doi.org/10.1088/1748-0221/10/12/C12031

[16] Mase, A., and Kawahata, K., "Fundamentals of Plasma Diagnostics with Electromagnetic Waves,” Journal of Plasma Fusion Research, Vol. 87, No. 5, May 2011, pp. 315-325, (in Japanese).

[17] Kohagura, J., Yoshikawa, M., Wang, X., Kuwahara, D., Ito, N., Nagayama, Y, Shima, Y., Nojiri, K., Sakamoto, M., Nakashima, Y., and Mase, A., “A 60-GHz Interferometery with a Local Oscillator Integrated Antenna Array for Divertor Simulation Experiments on GAMMA 10/PDX,” Review of Scientific Instruments, Vol. 87, No. 11, 11 Nov. 2016, $11 \mathrm{E} 127$.

https://doi.org/10.1063/1.4961292

[18] Tsukishima, T., and Mase, A., "Plasma Diagnostics with Electromagnetic Waves," Journal of Plasma and Fusion Research, Vol. 52, No. 2, 20 Sep. 1984, pp. 129-162, (in Japanese).

https://doi.org/10.1585/jspf1958.52.129

[19] Boeuf, J. P., "Tutorial: Physics and Modeling of Hall thrusters," Journal of Applied Physics, Vol. 121, No. 1, 3 Jan. 2017, 011101.

https://doi.org/10.1063/1.4972269

[20] Fife, J. M., Martinez-Sanchez, M., and Szabo, J., “A Numerical Study of Low-Frequency Discharge Oscillations in Hall Thrusters," AIAA Paper 97-3052, 1997.

[21] Boeuf, J. P., and Garrigues, L., "Low Frequency Oscillation in a Stationary Plasma Thruster," Journal of Applied Physics, Vol. 84, No. 7, 1998, pp. 3541-3554.

https://doi.org/10.1063/1.368529

[22] Yamamoto, N., Komurasaki, K. and Arakawa, Y., "Discharge Current Oscillation in Hall Thrusters, " Journal of Propulsion and Power, Vol. 21, No. 5, pp. 870-876, 2005.

https://doi.org/10.2514/1.12759 